\title{
Estimation of Driver Lane Change Intention Based on the LSTM and Dempster-Shafer Evidence Theory
}

\author{
Zhi-Qiang Liu $(\mathbb{D}$, Man-Cai Peng $(\mathbb{D}$, and Yue-Chen Sun $(\mathbb{D}$ \\ School of Automobile and Traffic Engineering, Jiangsu University, Zhenjiang 212013, China \\ Correspondence should be addressed to Man-Cai Peng; alexpengmc@163.com
}

Received 20 March 2020; Revised 10 January 2021; Accepted 15 January 2021; Published 27 January 2021

Academic Editor: Maria Vittoria Corazza

Copyright (C) 2021 Zhi-Qiang Liu et al. This is an open access article distributed under the Creative Commons Attribution License, which permits unrestricted use, distribution, and reproduction in any medium, provided the original work is properly cited.

\begin{abstract}
Rapid and correct estimation of driver lane change intention plays an important role in the advanced driver assistance system (ADAS), which could make the driver improve the reliability of the ADAS system and help to decrease driver workload. In this study, a method based on the long short-term memory network (LSTM) and Dempster-Shafer evidence theory is proposed. The model consists of a preliminary decision-making label and a final decision-making label. Driver visual information, head orientation, and vehicle dynamics are collected by preliminary decision-making label. Then, LSTM is used to calculate the initial probability of the driver lane change (left, right, and lane keeping) maneuver intention. The outputs of LSTM are normalized and assigned a basic probability by the Dempster-Shafer evidence theory. The final decision-making label analyzes the information and outputs the probability of each lane change intention and the decision is to identify the driver's current intention. The experimental results show that the accuracy of the model is $90.7 \%$ for the intention of changing left and $89.1 \%$ for the intention of changing right. The outcome of this work is an essential component for all levels of road vehicle automation.
\end{abstract}

\section{Introduction}

Traffic crashes and collisions worldwide become increasingly complicated in terms of safety with the number of vehicles increasing. Most of them should be attributed to drivers, such as operation errors, judgment mistakes, and cognitive overload [1-3]. Drivers have an essential impact on the road environment and traffic context by performing vehicles according to local traffic laws and their driving willingness. It is a dynamic system consisting of traffic, driver, and vehicle because they influence and interact with each other dynamically [4]. Since drivers are one of the parts of the loop, a thorough understanding of driver behaviors, especially lane changing maneuver intention, would help effectively improve driving safety and reduce traffic accidents $[5,6]$. Advanced driver assistance systems (ADAS) are intended to enhance driver performance and improve transportation safety. Nowadays, many ADAS products, such as adaptive cruise control [7], side warning assistance system [8], and lane departure warning system [9] serve to provide traffic context for drivers. All the products interact with human drivers passively because they fail to understand driving performance rapidly. Hence, the next generation of ADAS products are expected for dynamic interaction and mutual understanding between intelligent units and drivers' behavior $[10,11]$.

This paper aims to propose a framework to estimate drivers' lane change maneuver intention based on naturalistic driving data. Understanding and inferring drivers' intention has a positive effect on the development of ADAS [12]. The method can improve the active safety of drivers and provide surrendering traffic context information for drivers. Estimating driver intention can infer their behaviors at an early stage or before maneuvers happens after maneuvers are initiated. ADAS can generate appropriate notification and assistance to drivers with the identification of drivers' intention accurately [13] or warn of the possible dangers based on drivers' interested regions and future trajectories [14]. Berndt [15] showed that only $64 \%$ of drivers turn on the signal when changing lanes, and only $50 \%$ of them turn on the signal before starting to change lanes. A report by the national highway traffic safety administration (NHTSA) also 
pointed out that by counting 667 lane changes, the turn-on rate of the signal for left and right lane changes was $48 \%$ and $35 \%$, respectively [16]. The low turn-on rate of the signal and the untimely turn-on phenomenon will cause this type of system to fail to identify the driver's intention in a timely and effective manner. Therefore, it is necessary to recognize the driver's intention through other characteristics. Studies have shown that compared to lane keeping, drivers will show unique eye movements before changing lanes, and vehicle operating state parameters will also show obvious differences [17]. As the most unstable main factor in the human-vehicleroad closed-loop system, drivers often exhibit different driving characteristics during driving. For example, drivers will adopt different processing methods in the choice of lane changing timing and the search of surrounding traffic context information with regard to lane changing behavior.

Intelligent vehicles are expected to understand drivers' intention mechanisms and predict possible dangers and regions of interest, which also could be used to design human-like decision-making and behavior algorithms [18]. Although human drivers are not one of the components of completely autonomous vehicles, this does not mean the vehicle is not necessary to perceive the surrendering information, make a decision, and act like human drivers. It is a long-term task for intelligent vehicles since the rule-based methods can not adequately meet the requirements of the various and uncertain situations in the real world to learn how and why human drivers generate intention and make decisions. In the future, vehicle automation is expected to be more efficient based on data-driven intention and decisionmaking models [19].

It is well known that inferring driver lane change maneuver intention is considered a promising technology. But a few commercial products have been applied in automobile manufacturers because the development of estimating driver intention is still challenging, and the accuracy and robustness need further analysis and improvement. This paper proposes a double layer framework, consisting of a preliminary decision-making label and final decision-making label, to improve the accuracy of lane change maneuver intention identification. LSTM is utilized in the preliminary label to deal with time-series driving sequence and the Dempster-Shafer evidence theory is used in the final label to manage the uncertain lane change maneuver intention. LSTM is a popular model and has achieved a large amount of regression, classification, and prediction [20, 21]. The Dempster-Shafer evidence theory is an uncertain reasoning method, which satisfies weaker conditions than the Bayesian probability theory and has the ability to directly express the uncertain and unknown $[22,23]$. The reasons for adopting the LSTM and Dempster-Shafer evidence theory to estimate drivers' intention are multiple. Firstly, a great majority of the existing lane change maneuver intention detection algorithms based on machine learning are easily affected by training data. When the training data changes, different weight sets and parameter sets can be generated. LSTM improves the robustness and accuracy of the model by introducing different networks [24]. Secondly, the Dempster-Shafer evidence theory needs weaker conditions than the Bayesian theory of probability, so it is often regarded as an extension of the Bayesian theory. The probability assigned to each subset is limited by a lower bound and an upper bound, which, respectively, measure the total belief and the total plausibility for the objects in the subset. Furthermore, the Dempster-Shafer evidence theory has the ability to combine pairs of evidence or belief functions to derive new evidence or belief function. Due to its ability to handle the uncertainty or imprecision in the evidence, the Dempster-Shafer theory has been widely applied in recent years $[25,26]$.

Driver intention has been widely studied in the past two decades. Previous studies related to driver lane change intention have focused on ramp $[27,28]$, intersection $[29,30]$, and highway [31,32] and many methodologies have been proposed. For merging different categories of behavior, a classification regression tree was created and trained [27]. For the ramp entrance of the freeway, the Bayesian theorem to obtain the probability of driving intention was applied [28]. The hidden Markov model is trained for individual driving behavior [29], which is used to predict future driving behavior, while the support vector machine is proposed [30]. For the prediction of highway lane change behavior, the situation and motion based on features are implemented to calculate the individual probability of lane change behavior and combine them as the final prediction [31]. Support vector machine and Bayesian filter are used to identify lane change intention [32]. A method for predicting lane change of freeway is proposed by combining support vector machine with artificial neural network classifier [33]. Instead of detecting maneuver patterns of driving behaviors, the determination and classification of driving style have been investigated [34].

Nowadays, it is widely recognized that an accurate estimation of the lane change maneuver intention system should rely on a holistic method, which needs to fuse the multimodal data in the traffic-driver-vehicle loop [35]. Hence, more signals in the loop should be collected to recognize the driver intention. In terms of traffic, the most widely used features are lane signs, location of surrounding vehicles, global positioning system, and digital maps [36-38]. Major driver behavioral features include head orientation and motion, eye information, and body gestures $[39,40]$. Vehicle status signals can be collected from can bus/ Ethernet in the vehicle, including vehicle speed, acceleration, steering wheel angle, steering signal, brake, and pedal $[12,41]$.

In spite of previous studies that achieved some successful cases in the estimation of lane change maneuver intention, few works have been devoted to taking both sequential lane change maneuver behavior and uncertain intention recognition into consideration. This paper proposes a system framework for the identification and prediction of drivers' lane change maneuver intention on the highway. The method is developed to efficiently estimate driver and vehicle characteristic parameters by modeling the eye information, head orientation, and vehicle dynamics. A neural network with LSTM layer is trained to handle sequential lane change behavior and the Dempster-Shafer evidence theory 
is adopted to deal with uncertain intention recognition with the parameterized driving characteristics for predicting driver behavior transitions. The designed system has been tested and verified by real-world traffic data and the results indicate the accuracy of estimating lane change maneuver intention is high. Overall, the proposed system is useful for assisting an intelligent vehicle's decision-making process in naturalistic driving with its high-quality predictions.

\section{Preliminary Study}

Initialization lane change intention, preparation lane change, and action lane change behavior make up a complete lane change maneuver process, and the estimation and prediction of the driver intention primarily focus on the first part [42]. Initial lane change intention is the result of drivers' perception and evaluation of the surrounding environment and traffic context conditions. Drivers will produce the intention if the external conditions satisfy both available target lane and driving requirements. Before performing lane change maneuver, there is a corresponding change in the driver eye information and head motion [43], and vehicle dynamics and position in the lane will also change when the driver starts the operation of lane change maneuver [44]. Hence, characteristic parameters come into being during producing and performing lane change maneuver behavior which can be detected and will change in a specific way.

2.1. Analysis of Drivers' Behavior. Driving intention is a kind of cogitation and inner state that the driver receives external information and decides what driving behavior to perform $[12,20]$. The executive process of driving behavior is completed by the driver's sense, perception, and other organs according to a certain time series [45]. Drivers will first perceive and make decisions on the current road traffic context information to form a certain driving intention during driving. After intention is generated, the driver needs to comprehensively consider his own subjective desire such as destination, driving experience, and operating habits to further guide the formation of driving intention [46].

The ultimate purpose of driving intention is to provide guidance for the driver to carry out subsequent driving behavior so that the vehicle can drive safely according to their own wishes. At the same time, the change in the vehicle state also reflects the driver's intention. The driver can change the vehicle state through different operation combinations, which means the driving behavior of the vehicle is diversified under the guidance of the same driving intention. The specific operation combination is determined by the current actual road traffic elements and the driver's driving habits. Drivers will evaluate the present traffic context information before initialization lane change intention. Drivers will generate lane change intention if the target lane is available and the current lane is in a traffic jam.

2.2. Analysis of Driver Eye Information. It is necessary to analyze drivers' eye characteristic parameters because most information is obtained by eyes during driving [43]. The driver's eye movement data is collected from $5 \mathrm{~s}$ before lane change maneuver. Figure 1 shows the curve of the driver's eye scanning angle in horizontal direction changing with the time under the condition of lane change left (LCL), lane change right (LCR), and lane keeping (LK). It can be seen that the driver's scanning angle fluctuates obviously when changing lanes, and there are obvious differences in scanning angles under different states. Therefore, the scanning angle can be used as one of the parameters to identify the driver lane change intention.

Figure 2 shows the boxplot of the saccadic velocity at different stages. It can be observed that the saccadic velocity of drivers is the LCR $>\mathrm{LCL}>\mathrm{LK}$, corresponding to different intentions of lane changing. When the significance level shown in Table 1 is $\alpha=0.05$, there is no abnormality in the $t$ test results of the driver's saccade velocity. Because there is a great difference between the saccade velocity when changing lane left and right, this paper takes the saccade velocity as one of the parameters to identify the driver's lane change intention.

Drivers need to turn their heads to get more information during driving because of limited visual angle. Research shows that the head movement often precedes the eye movement in the process of getting more vision [13]. Therefore, head movement is also an essential factor in the visual analysis of drivers. Because there are many position parameters about head movement, this paper selects horizontal and vertical rotation parameters for analysis. Figure 3 is the statistical chart of the head horizontal corner box corresponding to different driving behaviors. It can be observed that the horizontal corner of drivers is the LCR $>$ LCL $>$ LK. When the data shown in Table 2 is $\alpha=0.05$, it is the $t$-test result of data of the driver head motion, among which the upper triangle is the $t$-test result of the vertical corner of the driver's head, and the lower triangle is the $t$-test result of the horizontal corner of the driver's head.

The results indicate that there is a significant difference in the horizontal corner of the driver's head in each lane change intention, and the $t$-test result of the vertical corner of the driver's head is abnormal. There is no significant difference in the vertical corner of the driver's head under the three states of intentions of lane changing. Therefore, this paper only selects the horizontal corner of the driver's head as the parameter to judge the intention of lane changing of the driver (* indicates that the test is abnormal).

2.3. Analysis of Vehicle Dynamics. The model will achieve a highly accurate estimation when the steering wheel angle and the vehicle longitudinal acceleration are used as the characteristic parameters [47]. The longitudinal acceleration is the description of the driver's expected speed, and the steering wheel angle directly reflects the operation of the driver lane change behavior.

The driver can change lanes by turning the steering wheel continuously and greatly during driving. Steering wheel angle is a common feature parameter in lane change intention recognition [28]. Berndt et al. [15] showed that only $64 \%$ of drivers turn on the signal when changing lanes, 


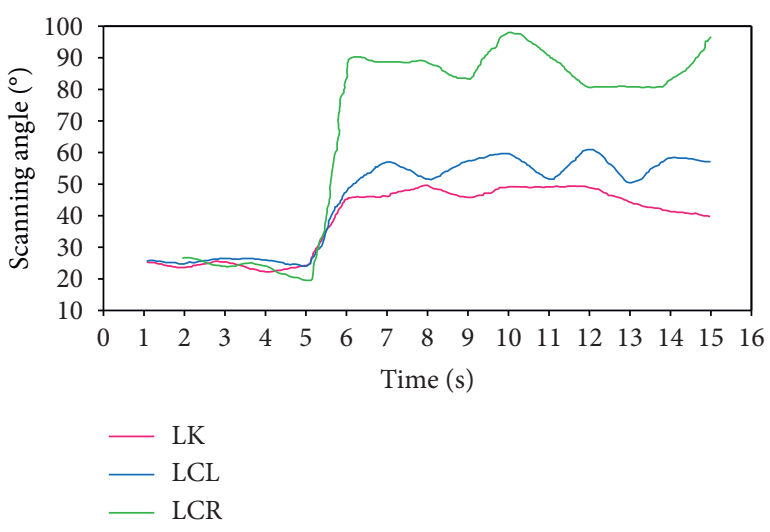

FIGURE 1: Driver scanning angle under different driving intentions.

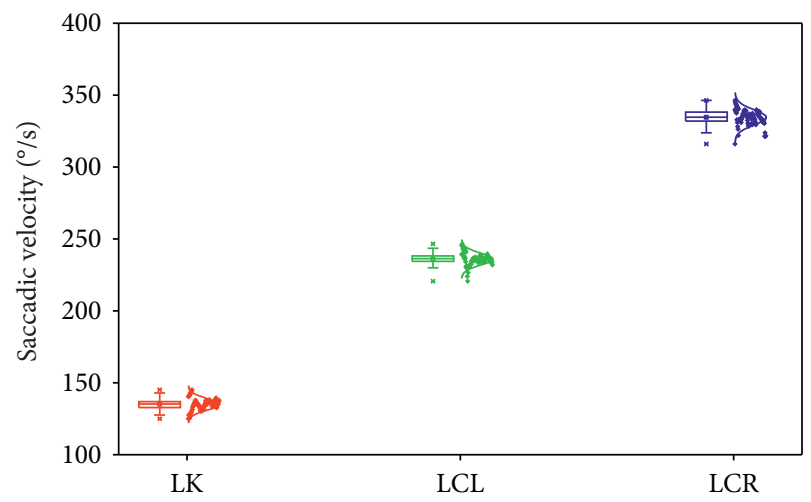

FIGURE 2: The boxplot of saccadic velocity under different driving intentions.

TABLE 1: $t$-test results of driver scanning speed.

\begin{tabular}{lccc}
\hline Intention & LK & LCL & LCR \\
\hline LK & - & - & - \\
LCL & 0.01 & - & - \\
LCR & 0.015 & 0.03 & - \\
\hline
\end{tabular}

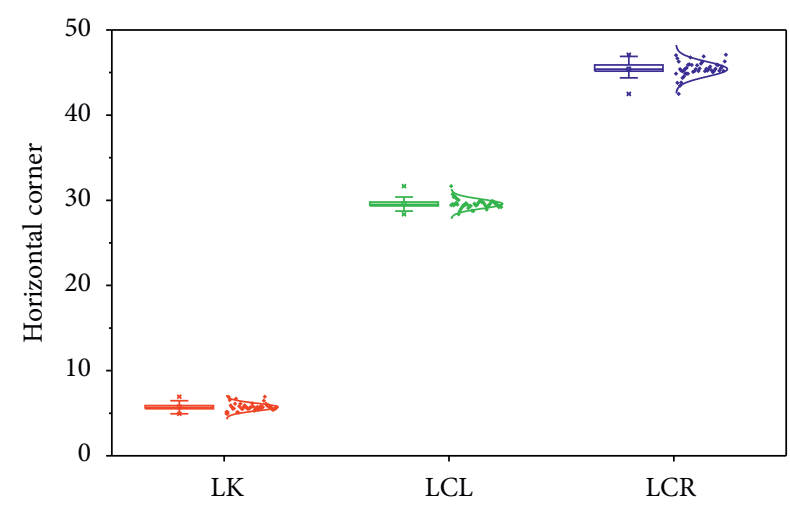

FIGURE 3: The boxplot of the horizontal corner of the driver's head under different driving intentions.
TABLE 2: $t$-test results of the horizontal and vertical angle of the driver's head.

\begin{tabular}{lccc}
\hline Intention & LK & LCL & LCR \\
\hline LK & - & 0.034 & 0.022 \\
LCL & 0.017 & - & $0.068^{*}$ \\
LCR & 0.013 & 0.007 & - \\
\hline
\end{tabular}

and only $50 \%$ of them turn on the signal before starting to change lanes. The efficiency of lane change intention recognition is easily affected by turning on the signal rate low or not turning on in time. Hence, the turn signal is used as one of the parameters to identify lane changing intention in this study. Input signal 0 indicates that the turn signal is off, and 1 indicates that the turn signal is on. The deviation between vehicle and lane centerline reflects the lateral displacement of the vehicle during lane changing behavior, which can be used as the characteristic index of the road environment. As shown in Figure 4, it is the statistical chart of self-vehicle and lane centerline.

\section{Model Building}

3.1. Recurrent Neural Network. Recognization lane changing of driver intention is a continuous process with dynamic behavior fluctuations over time. Most of the classification algorithms adopted in previous studies focused on the characteristic variables reflecting the whole sequence, but it was difficult to capture the change of the process of driver behavior. Considering the time dependence of lane changing, the driver status can be identified by using the complete time-series information. In recent years, deep learning has shown great potential in dealing with multivariate time-series classification. The recurrent neural network is a kind of artificial neural network in which nodes are connected in a ring directionally. The internal state of this network can show the dynamic behavior. Different from the feedforward neural network, the recurrent neural network can use internal memory to process sequence data, which makes it often utilized to deal with some complex deep learning problems. However, due to the gradient vanishing effect, the simple recurrent neural network cannot deal with the long sequence dependence well. In order to overcome this limitation, Hochreiter and Schmidhuber proposed a long short-term memory network (LSTM), as shown in Figure 5 [48]. Each hidden unit in LSTM contains one or more memory cells. The operation of these memory cells is controlled by the internal input gate $i_{t}$, the forgetting gate $f_{t}$, and the output gate $o_{t}$. The input gate determines which part of the input value can be used to update the memory state, the forgetting gate determines the information to be retained or removed in the memory unit, and the output gate determines the output content. The expression of the three gates at time $t$ can be expressed by the following formula: Input gate:

$$
i_{t}=\sigma\left[W_{i}\left(x_{t}+h_{t-1}+C_{t-1}\right)+b_{i}\right] .
$$




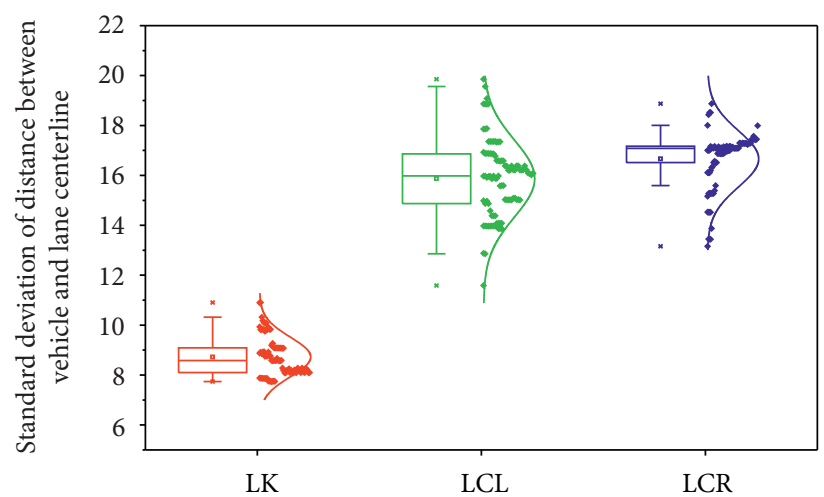

FIgURE 4: Standard deviation of the distance between the vehicle and lane centerline.

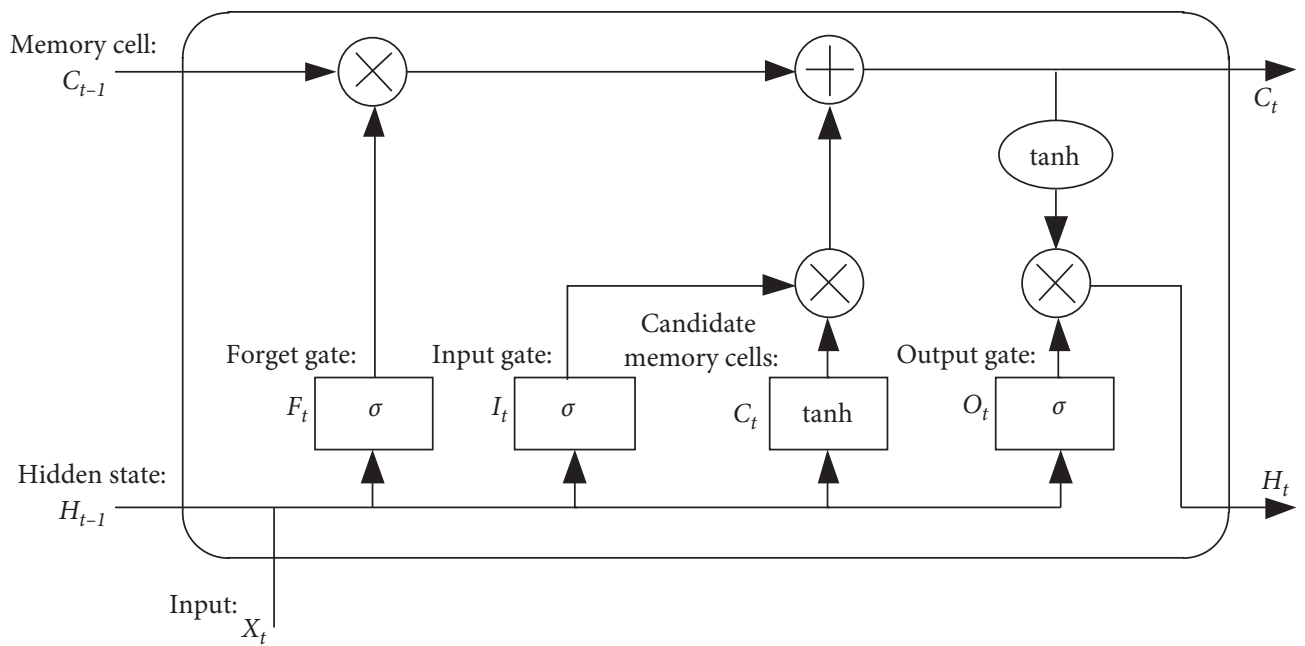

FIgURE 5: Structure of long short-term memory.

Forget gate:

$$
\begin{aligned}
& f_{t}=\sigma\left[W_{f}\left(x_{t}+h_{t-1}+C_{t-1}\right)+b_{f}\right], \\
& \overline{C_{t}}=\tanh \left[W_{c}\left(x_{t}+h_{t-1}\right)+b_{c}\right] .
\end{aligned}
$$

Memory cell unit:

$$
C_{t}=f_{t} \times C_{t-1}+i_{t} \times \overline{C_{t}} .
$$

Output gate:

$$
\begin{aligned}
& o_{t}=\sigma\left[W_{o}\left(x_{t}+h_{t-1}+C_{t-1}\right)+b_{o}\right], \\
& h_{t}=o_{t} \times \tanh \left(C_{t}\right),
\end{aligned}
$$

where $i_{t}, f_{t}$, and $o_{t}$ are the score of the input gate, forgetting gate, and output gate passing through activation function at time $t . W_{i}, W_{f}, W_{c}, W_{o}, W_{f}$ and $b_{i}, b_{f}, b_{c}, b_{o}, b_{f}$ are weight and deviation. $C_{t}$ is an alternative value of memory cell state $C_{t}$ at time $t$. $x_{t}$ and $h_{t}$ are the input of memory cell and the final category output. At each time step, the LSTM can read, write, or reset the memory unit through these three gates. The strategy allows LSTM to memorize and access the information before the multistep. The input gate, output gate, and forget gate correspond to the operation of writing, reading, and updating the historical state of the driver's lane changing intention characteristic parameter sequence.

3.2. LSTM Realizes the Dempster-Shafer Evidence Theory Basic Probability Assignment. LSTM is mainly used to process and predict sequence data, and its source is to describe the relationship between the current output of a sequence and the previous information. From the network structure, LSTM will memorize the previous information and combine with the present input; it can output the probability values of various intentions at the next time through a softmax classifier. To predict the output at time $t$, the memory of time $t$ is obtained by using the memory of time $t-1$ and the input of time $t$, and then the probabilities of various intentions are output by the softmax classifier:

$$
\begin{aligned}
P\left(y_{t}\right) & =\frac{e^{Z_{j}}}{\sum_{1}^{k} e^{Z_{k}}}, \quad(k=3), \\
Z & =X^{T} W_{j},
\end{aligned}
$$

where $X$ is the sample matrix and $j(j=1,2,3)$ is the number of driving intentions. 
The recurrent neural network is adopted to solve the basic probability assignment problem of evidence theory. The designed structure of LSTM is as follows: seven characteristic parameters are selected, which are driver's scanning angle, scanning velocity, head horizontal angle, vehicle longitudinal acceleration, steering wheel angle, turn signal, and standard deviation of lane position. The input number of nodes of the neural network is 7 , and the hidden layer nodes number is set to be adjustable. The number of lane changing intention to be identified is 3 , so the number of output layer nodes is 3 . Lane change left corresponds to the output is $[1,0,0]$, lane change right corresponds to the output is $[0,1,0]$, and lane change keeping corresponds to the output is $[0,0,1]$.

In order to reduce the mistakes on the basis of keeping the relationship existing in the original data, this study adopts the deviation standardization method to normalize the original data, and the conversion formula is

$$
X^{*}=\frac{X-X_{\min }}{X_{\max }-X_{\min }},
$$

where $X^{*}$ is the data after normalization, $X$ is the original data, $X_{\min }$ is the minimum value of data in the initial sample, $X_{\max }$ is the maximum value of data in the initial sample.

3.3. Recognition Algorithm. The output of LSTM after training is normalized as the basic probability distribution of each focal element. Figure 6 is the framework of lane change intention based on LSTM and evidence theory. The characteristic parameter matrix, which represents different lane change intention, is input into the trained recurrent neural network, and the probability distribution of each intention is initially output through the softmax layer. In order to get a piece of evidence within a certain time threshold, the normalized output of LSTM is taken as the basic probability allocation of evidence theory. First, the appropriate conflict coefficient threshold is set according to the specific using environment. Then, the evidence conflict processing method based on the evidence distance is utilized to deal with the evidence conflict within the time threshold, and the conflict probability of the focus element with high priority is assigned, so as to improve the reliability and accuracy of the final fusion results. Finally, the basic probability distribution function is used as the decision, and the Dempster synthesis rule is adopted to fuse the evidence within the time threshold to output the probability of each lane changing intention.

\section{Experimental Analysis}

4.1. Experimental Environment and Data. Simulation experiments about lane change left, lane change right, and lane keeping have been carried to verify the validity and reliability of the proposed framework. RNN-LSTM is built up by TensorFlow and some concrete parameters are as follows: the experimental platform is based on the upper framework Keras of TensorFlow version_1.1, and the experimental programming language is Python 3.7. The original data of eye information and vehicle dynamics are separately collected by smart eye protracker and six-degree of freedom from Jiangsu University. The experimental scene is designed as a two-way six-lane expressway with a length of $100 \mathrm{~m}$ and a width of $3.5 \mathrm{~m}$. The host controller unit of the driving simulator is used to synchronously collect the longitudinal acceleration, steering wheel angle, and the distance between the vehicle and the centerline of the lane. Meanwhile, the system of smart eyes racker is used to collect the data of driver eye movement and head motion, as shown in Figure 7.

4.2. Estimation Model. Figure 8 is the flow chart of model simulation. First, the training data is normalized by the deviation, and different hidden layer neuron nodes are set. Then, the continuous lane change behavior data of $10 \mathrm{~s}$ are taken as the input of the network, and the driving behavior data of the next moment predicted by LSTM is taken as the output of the initial decision. The output of the Dempster-Shafer decision layer is regarded as the final decision output. In the experiment, by comparing the mean square error different hidden layer, the mean square error diagram of the neural network under different numbers of hidden layer neuron nodes in the experiment process is shown in Figure 9. When the number of neuron nodes in the LSTM layer is 11 , the mean square error of the neural network is the smallest. Therefore, the number of neuron nodes in the LSTM layer is set as 11 .

4.3. Comparison between Models. In this paper, the backpropagation and Dempster-Shafer (BP-DS) algorithm is used as a controlled experiment. The concrete parameters are as follows: the number of backpropagation (BP) neural network layers is designed as 3, and the number of input nodes is set as 7. The number of hidden layer nodes is set as adjustable, and the hidden activation function layer is set as a hyperbolic tangent function. The target error is set to 10-6, the learning rate is 0.05 , the maximum training times is 3000 , and the number of nodes in the output layer is 3 . Lane change left corresponding to the output is $[1,0,0]$, lane change right corresponding to the output is $[0,1,0]$, and lane change keeping corresponding to the output is $[0,0,1]$.

In the LSTM-DS model, the input layer of the LSTM network model is selected as 3, which means that the data input scale for training and testing is a matrix of $3 \times 7$, corresponding to the first three measured driving operations. The number of nodes in the LSTM layer is 11, and the output through the softmax layer is the probability corresponding to various intentions. Set the target error as 10-6, the learning rate as 0.05 , and the maximum training times as 3000. After LSTM-DS and BP-DS training, the same tested samples of lane change maneuver behavior in three times, the prediction results of five consecutive times are selected to compare the mean square error of the two models as shown in Figure 10. It can be seen from Figure 10 that the mean square error of BP-DS is always greater than that of LSTMDS, and the growth rate of the mean square error of BP-DS is much faster than LSTM-DS. It can be concluded that the recognition accuracy of LSTM-DS is higher than that of BPDS. 


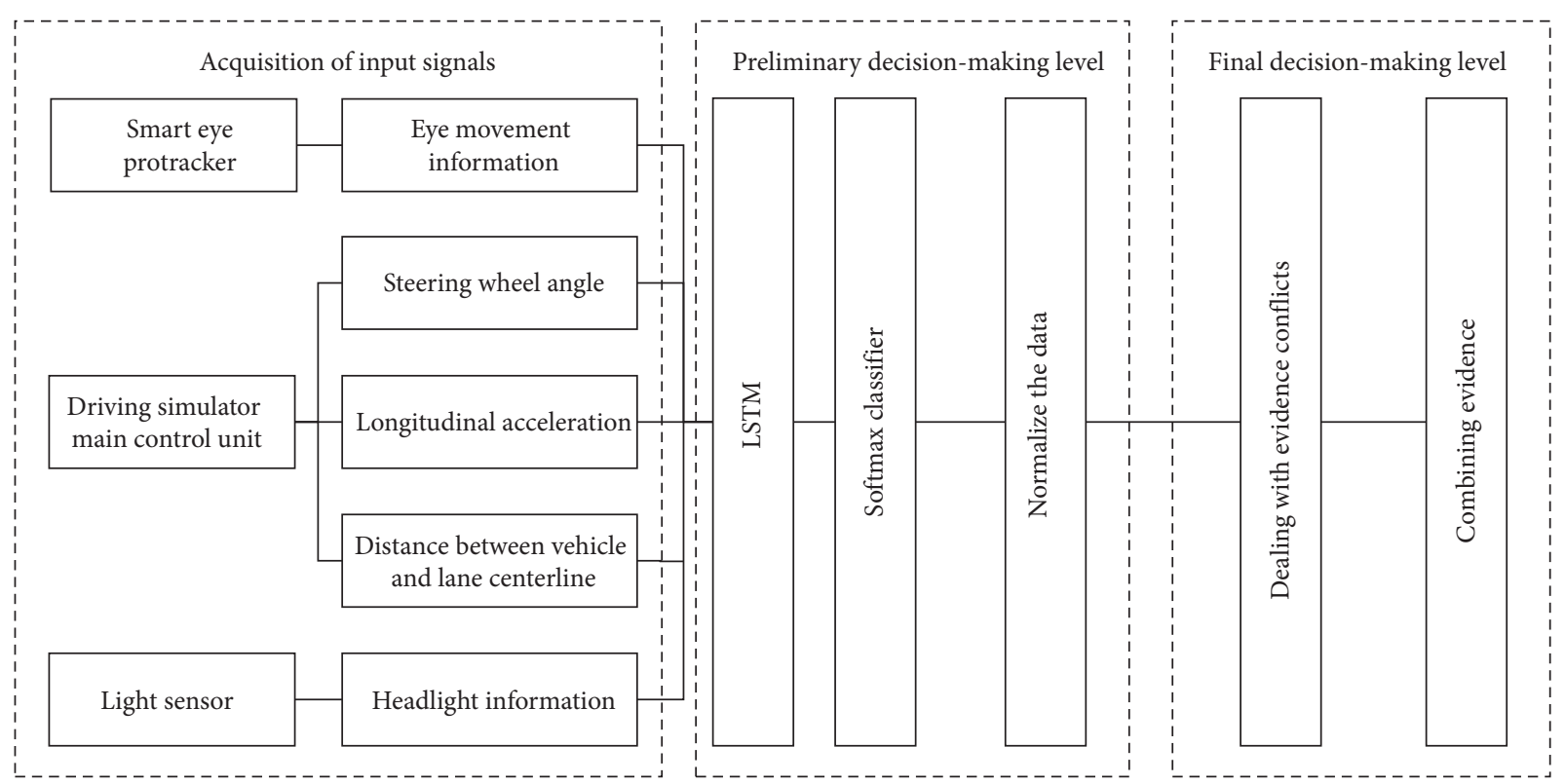

FIGURE 6: Framework of identification lane change intention.

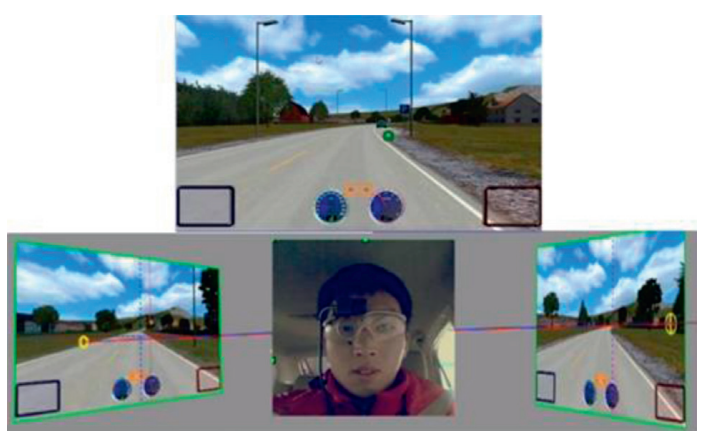

FIGURE 7: Driving simulator and eye tracker system.

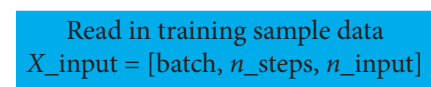

steps, $n$ _input]
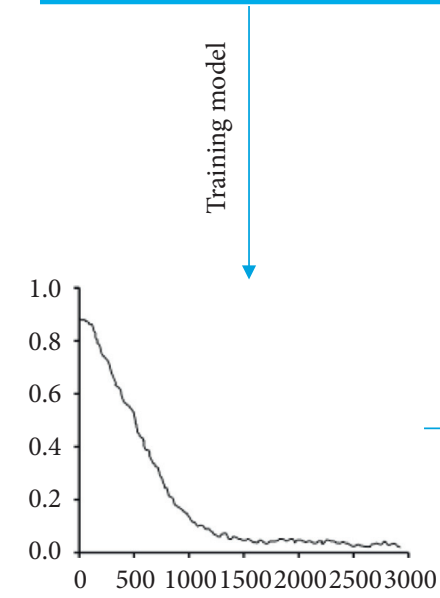

FIGURE 8: Flow chart of the simulation program of the model. 


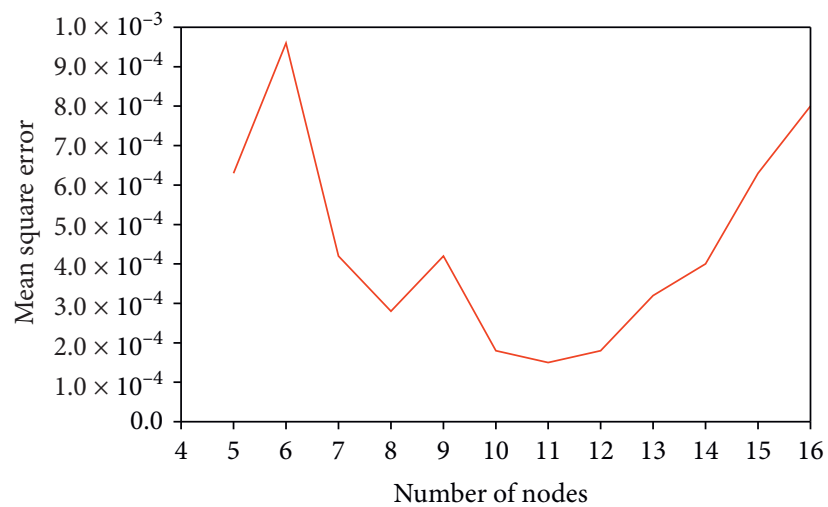

FIGURE 9: Mean square error of the neural network under different hidden layer neuron nodes.

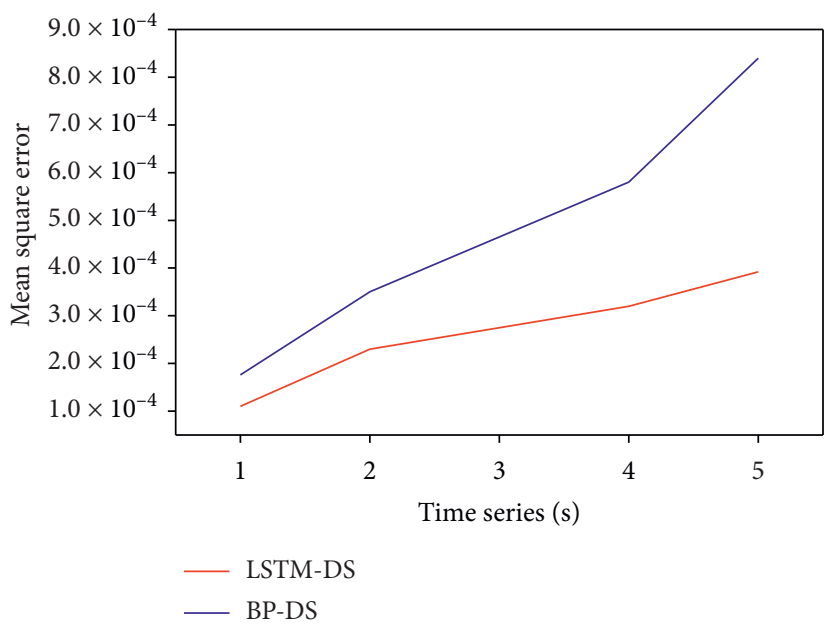

Figure 10: Mean square error curve of the models.

4.4. Model Prediction. In the experiment, 200 groups of data are selected as the original data (185 groups as the training data and the other 15 groups as the test data) and the training samples are input into the neural network to train the recurrent neural network by the backpropagation algorithm. After 2921 training times, the number of hidden layer nodes of the recurrent neural network is 11 , and the output target error is 106. Then, input the test sample data to the trained LSTM and take the normalized output of LSTM as the basic probability distribution of evidence theory. The recognition accuracy of the model for real-time collected data is shown in Table 3.

By calculating the accuracy of model recognition (the proportion of correctly identified samples to the total number of test samples), the recognition of lane change left intention is $90.7 \%$, the recognition of lane change right intention is $89.1 \%$, and the recognition of lane keeping intention is $85.63 \%$. The accuracy of lane keeping is lower than the left and right lane change, which is caused by the large vibration of some data such as steering wheel angle.

The recognition result of the driver's intention to change lanes will have a direct impact on the efficiency of subsequent trajectory prediction. Therefore, this paper selects precision, recall, F1-score, and accuracy as the evaluation indicators. The definitions of each indicator are as follows: precision refers to recognition of the number of correct positive samples accounting for the proportion of the number of positive samples judged by the model. The recall rate refers to the proportion of the correct positive sample format to the actual number of positive samples. F1-score is the harmonic average of the precision rate and the recall rate. The accuracy rate is the ratio of the number of correct identifications to the total number of samples. Table 4 shows 
TABLE 3: Part of the test results.

\begin{tabular}{|c|c|c|c|}
\hline $\begin{array}{l}\text { Real } \\
\text { intention }\end{array}$ & Output of LSTM & $\begin{array}{c}\text { Output of } \\
\text { DS }\end{array}$ & $\begin{array}{c}\text { Decision } \\
\text { results }\end{array}$ \\
\hline LCL & $\begin{array}{c}0.9223,0.0423 \\
0.0295] \\
{[0.9043,0.0299} \\
0.0435] \\
{[0.9148,0.0395} \\
0.0457] \\
{[0.9245,0.0382} \\
0.0298] \\
{[0.9127,0.0467} \\
0.0395] \\
{[0.9119,0.0397} \\
0.0484] \\
{[0.9565,0.0458} \\
0.0295]\end{array}$ & {$[1,0,0]$} & LCL \\
\hline LCR & $\begin{array}{c}0.0373,0.9533 \\
0.0203] \\
{[0.0265,0.9144} \\
0.0587] \\
{[0.0229,0.9236} \\
0.0439] \\
{[0.0174,0.9502} \\
0.0324] \\
{[0.0225,0.9443} \\
0.0336] \\
\end{array}$ & {$[0,1,0]$} & LCR \\
\hline LK & $\begin{array}{c}{[0.0342,0.0175} \\
0.9483] \\
{[0.0463,0.0224} \\
0.9313] \\
{[0.0337,0.0276} \\
0.9387]\end{array}$ & {$[0,0,1]$} & LK \\
\hline
\end{tabular}

TABLE 4: Recognition results of driver lane changing based on test data.

\begin{tabular}{lcccc}
\hline Lane changing & Precision & Recall & F1-score & Accuracy \\
\hline LCL & 0.907 & 0.875 & 0.891 & \\
LK & 0.856 & 0.861 & 0.858 & 0.869 \\
LCR & 0.891 & 0.865 & 0.878 & \\
\hline
\end{tabular}

the results of testing the intention recognition model with the data in the test set.

\section{Conclusion}

This paper proposes a system framework for the estimation and prediction of lane change maneuver intention on the highway. A method with LSTM and evidence theory technique is developed to efficiently identify characteristic parameters by modeling the drivers' eye information, head motion, and vehicle dynamics; a neural network with LSTM layer is trained to utilize the parameterized driving characteristics for time-series sequence by training sets data. Evidence theory is used to deal with evidence conflicts and output the final drivers' intention. Validation results by the test sets data prove that the designed framework is capable of recognizing and inferring lane change maneuver intention with high accuracy. Due to the recognition framework trained based on the data collected by the driving simulator, the actual traffic scene will be more complex, so the model will be limited in the application scene.

This paper reveals the changing laws of vehicle dynamic parameters and drivers' visual characteristics in typical highway driving behaviors, and enrich relevant theoretical studies on driving behaviors such as lane changing and lane keeping. At the same time, it provides a certain theoretical basis for the recognition of driver intention in other road scenes. By realizing the early recognition of the driver's intention to change lanes, it serves the active safety warning system. By predicting behaviors such as lane changing and following, it further improves the system services such as lane changing assistance and lane keeping assistance for drivers, reducing the burden on the driver and also allowing driving intervention in emergency situations and avoiding accidents.

In summary, the in-depth study of methods and theories for the recognition of highway drivers' lane change maneuver intentions can provide theoretical and technical support for the research and application of ADAS and related intelligent vehicles, thereby improving the safety and comfort of drivers and improve the traffic environment. The next step is to improve the accuracy of the model to identify and further improve the application of the model on urban roads.

\section{Data Availability}

All the data included in this study are available upon request by contact with the corresponding author.

\section{Conflicts of Interest}

The authors declare that they have no conflicts of interest related to this work.

\section{Acknowledgments}

This research was supported by National Natural Science Foundation of China (61403172). The authors also wish to thank all the participants, school administrators, and the local government who helped facilitate their intervention work.

\section{References}

[1] S. A. Birrell, D. Wilson, C. P. Yang, G. Dhadyalla, and P. Jennings, "How driver behaviour and parking alignment affects inductive charging systems for electric vehicles," Transportation Research Part C: Emerging Technologies, vol. 58, pp. 721-731, 2015.

[2] E. Bellis and J. Page, National Motor Vehicle Crash Causation Survey ( NMVCCS ) SAS Analytical Users Manual, National Highway Traffic Safety Administration, Washington, DC, USA, 2008.

[3] E. Ohn-Bar and M. M. Trivedi, "Looking at humans in the age of self-driving and highly automated vehicles," IEEE Transactions on Intelligent Vehicles, vol. 1, no. 1, pp. 90-104, 2016. 
[4] J. Barcelo, Fundamentals of Traffic Simulation, Springer, Berlin, Germany, 2010.

[5] Y. Xing, C. Lv, H. Wang, D. Cao, E. Velenis, and F. Y. Wang, "Driver activity recognition for intelligent vehicles: a deep learning approach," IEEE Transactions on Vehicular Technology, vol. 68, no. 6, pp. 5379-5390, 2019.

[6] C. Lv, H. Wang, D. Cao et al., "Characterization of driver neuromuscular dynamics for human-automation collaboration design of automated vehicles," IEEE/ASME Transactions on Mechatronics, vol. 23, no. 6, pp. 2558-2567, 2018.

[7] V. Milanes, S. E. Shladover, J. Spring, C. Nowakowski, H. Kawazoe, and M. Nakamura, "Cooperative adaptive cruise control in real traffic situations," IEEE Transactions on Intelligent Transportation Systems, vol. 15, no. 1, pp. 296-305, 2014.

[8] E. Adell, A. Várhelyi, and M. dalla Fontana, "The effects of a driver assistance system for safe speed and safe distance-a real-life field study," Transportation Research Part C: Emerging Technologies, vol. 19, no. 1, pp. 145-155, 2011.

[9] Y. Xing, C. Lv, L. Chen et al., "Advances in vision-based lane detection: algorithms, integration, assessment, and perspectives on ACP-based parallel vision," IEEE/CAA Journal of Automatica Sinica, vol. 5, no. 3, pp. 645-661, 2018.

[10] D. Tran, J. Du, W. Sheng, D. Osipychev, Y. Sun, and H. Bai, “A human-vehicle collaborative driving framework for driver assistance," IEEE Transactions on Intelligent Transportation Systems, vol. 20, no. 9, pp. 3470-3485, 2019.

[11] P. Zhang and Z. Qian, "Managing traffic with raffles," Transportation Research Part C: Emerging Technologies, vol. 107, pp. 490-509, 2019.

[12] Y. Xing, C. Lv, H. Wang et al., "Driver lane change intention inference for intelligent vehicles: framework, survey, and challenges," IEEE Transactions on Vehicular Technology, vol. 68, no. 5, pp. 4377-4390, 2019.

[13] A. Doshi and M. M. Trivedi, "On the roles of eye gaze and head dynamics in predicting driver's intent to change lanes," IEEE Transactions on Intelligent Transportation Systems, vol. 10, no. 3, pp. 453-462, 2009.

[14] E. Ackerman, Drive.ai Brings Deep Learning to Self-Driving Cars, IEEE Spectrum, New York, NY, USA, 2016.

[15] H. Berndt, J. Emmert, and K. Dietmayer, "Continuous driver intention recognition with hidden markov models," in Proceedings of the IEEE Conference on Intelligent Transportation Systems, Proceedings, ITSC, Beijing, China, October 2008.

[16] S. E. Lee, E. C. B. Olsen, and W. W. Wierwille, "A comprehensive examination of naturalistic lane-changes," Virginia Tech Transportation Institute, Blacksburg, VA, USA, DOT HS 809 702, 2004.

[17] E. C. B. Olsen, "Modeling slow lead vehicle lane changing," Doctoral dissertations, Virginia Polytechnic Institute and State University, Blacksburg, VA, USA, 2003.

[18] A. Kuefler, J. Morton, T. Wheeler, and M. Kochenderfer, "Imitating driver behavior with generative adversarial networks," in Proceedings of the IEEE Intelligent Vehicles Symposium, Los Angeles, CA, USA, June 2017.

[19] L. Fridman, "Human-centered autonomous vehicle systems: principles of effective shared autonomy," 2018, https://arxiv. org/abs/1810.01835.

[20] A. Zyner, S. Worrall, J. Ward, and E. Nebot, "Long short term memory for driver intent prediction," in Proceedings of the IEEE Intelligent Vehicles Symposium, Proceedings, Los Angeles, CA, USA, June 2017.

[21] Y. Xing, C. Lv, H. Wang, D. Cao, and E. Velenis, "An ensemble deep learning approach for driver lane change intention inference," Transportation Research Part C: Emerging Technologies, vol. 115, p. 102615, 2020.

[22] P. Smets, "The transferable belief model and random sets," International Journal of Intelligent Systems, vol. 7, no. 1, pp. 37-46, 1992.

[23] D. Fixsen and R. P. S. Mahler, "The modified Dempster-Shafer approach to classification," IEEE Transactions on Systems, Man, and Cybernetics_Part A: Systems and Humans, vol. 27, no. 1, pp. 96-104, 1997.

[24] P. Izmailov, D. Podoprikhin, T. Garipov, D. Vetrov, and A. G. Wilson, "Averaging weights leads to wider optima and better generalization," in Proceedings of the 34th Conference on Uncertainty in Artificial Intelligence 2018, UAI 2018, Monterey, CA, USA, August 2018.

[25] B. Kang, Y. Deng, R. Sadiq, and S. Mahadevan, "Evidential cognitive maps," Knowledge-Based Systems, vol. 35, pp. 77-86, 2012.

[26] Y. Deng, W. Jiang, and R. Sadiq, "Modeling contaminant intrusion in water distribution networks: a new similaritybased DST method," Expert Systems with Applications, vol. 38, no. 1, pp. 571-578, 2011.

[27] Q. Meng and J. Weng, "Classification and regression tree approach for predicting drivers' merging behavior in shortterm work zone merging areas," Journal of Transportation Engineering, vol. 138, no. 8, pp. 1062-1070, 2012.

[28] C. Dong, J. M. Dolan, and B. Litkouhi, "Intention estimation for ramp merging control in autonomous driving," in Proceedings of the IEEE Intelligent Vehicles Symposium, Los Angeles, CA, USA, June 2017.

[29] V. Gadepally, A. Krishnamurthy, and U. Ozguner, "A framework for estimating driver decisions near intersections," IEEE Transactions on Intelligent Transportation Systems, vol. 15, no. 2, pp. 637-646, 2014.

[30] S. B. Amsalu, A. Homaifar, F. Afghah, S. Ramyar, and A. Kurt, "Driver behavior modeling near intersections using support vector machines based on statistical feature extraction," in Proceedings of the IEEE Intelligent Vehicles Symposium, Proceedings, Seoul, Republic of Korea, July 2015.

[31] C. Wissing, T. Nattermann, K. H. Glander, C. Hass, and T. Bertram, "Lane change prediction by combining movement and situation based probabilities," IFAC-PapersOnLine, vol. 50, no. 1, pp. 3554-3559, 2017.

[32] P. Kumar, M. Perrollaz, S. Lefevre, and C. Laugier, "Learningbased approach for online lane change intention prediction," in Proceedings of the IEEE Intelligent Vehicles Symposium, Proceedings, Gold Coast, Australia, June 2013.

[33] Y. Dou, F. Yan, and D. Feng, "Lane changing prediction at highway lane drops using support vector machine and artificial neural network classifiers," in Proceedings of the IEEE/ ASME International Conference on Advanced Intelligent Mechatronics, AIM, Banff, Canada, July 2016.

[34] O. Derbel and R. Landry, "Driving style assessment based on the GPS data and fuzzy inference systems," in Proceedings of the 12th International Multi-Conference on Systems, Signals and Devices, SSD 2015, Mahdia, Tunisia, March 2015.

[35] M. Liebner and F. Klanner, "Driver intent inference and risk assessment," in Handbook of Driver Assistance Systems: Basic Information, Components and Systems for Active Safety and ComfortSpringer, Dordrecht, Netherlands, 2015.

[36] X. Pi, W. Ma, and Z. Qian, "A general formulation for multimodal dynamic traffic assignment considering multi-class vehicles, public transit and parking," Transportation Research Part C: Emerging Technologies, vol. 104, pp. 369-389, 2019. 
[37] G. P. Gwon, W. S. Hur, S. W. Kim, and S. W. Seo, "Generation of a precise and efficient lane-level road map for intelligent vehicle systems," IEEE Transactions on Vehicular Technology, vol. 66, no. 6, pp. 4517-4533, 2017.

[38] Y. Wang, Y. Liu, H. Fujimoto, and Y. Hori, "Vision-based lateral state estimation for integrated control of automated vehicles considering multirate and unevenly delayed measurements," IEEE/ASME Transactions on Mechatronics, vol. 23, no. 6, pp. 2619-2627, 2018.

[39] C. Gou, Y. Wu, K. Wang, K. Wang, F. Y. Wang, and Q. Ji, “A joint cascaded framework for simultaneous eye detection and eye state estimation," Pattern Recognition, vol. 67, pp. 23-31, 2017.

[40] J. Chen and Q. Ji, "A probabilistic approach to online eye gaze tracking without explicit personal calibration," IEEE Transactions on Image Processing, vol. 24, no. 3, pp. 1076-1086, 2015.

[41] A. Doshi, B. T. Morris, and M. M. Trivedi, "On-road prediction of driver's intent with multimodal sensory cues," IEEE Pervasive Computing, vol. 10, no. 3, pp. 22-34, 2011.

[42] M. Keyvan-Ekbatani, V. L. Knoop, and W. Daamen, "Categorization of the lane change decision process on freeways," Transportation Research Part C: Emerging Technologies, vol. 69, pp. 515-526, 2016.

[43] Y. Ma, V. Havyarimana, J. Bai, and Z. Xiao, "Vision-based lane detection and lane-marking model inference: a three-step deep learning approach," in Proceedings of the International Symposium on Parallel Architectures, Algorithms and Programming, PAAP, Guangzhou, China, December 2018.

[44] R. Izquierdo, A. Quintanar, I. Parra, D. Fernandez-Llorca, and M. A. Sotelo, "Experimental validation of lane-change intention prediction methodologies based on CNN and LSTM," in Proceedings of the 2019 IEEE Intelligent Transportation Systems Conference (ITSC), Auckland, New Zealand, February 2019.

[45] A. Zyner, S. Worrall, and E. Nebot, "Naturalistic driver intention and path prediction using recurrent neural networks," IEEE Transactions on Intelligent Transportation Systems, vol. 21, no. 4, pp. 1584-1594, 2020.

[46] M. Bahram, C. Hubmann, A. Lawitzky, M. Aeberhard, and D. Wollherr, "A combined model- and learning-based framework for interaction-aware maneuver prediction," IEEE Transactions on Intelligent Transportation Systems, vol. 17, no. 6, pp. 1538-1550, 2016.

[47] N. Kuge, T. Yamamura, O. Shimoyama, and A. Liu, "A driver behavior recognition method based on a driver model framework," in Proceedings of the SAE, Dearborn, MI, USA, November 2000.

[48] S. Hochreiter and J. Schmidhuber, "Long short-term memory," Neural Computation, vol. 9, no. 8, pp. 1735-1780, 1997. 